\title{
Large Thoracolumbar Extradural Arachnoid Cyst Excised by Minimal Skipped Hemilaminectomy: A Case Report
}

\author{
Hwa Joong Lee, Won Ho Cho, In Ho Han, Byung Kwan Choi \\ Department of Neurosurgery and Medical Research Institute, Pusan National University Hospital, \\ Pusan National University School of Medicine, Busan, Korea
}

The standard treatment for symptomatic spinal extradural arachnoid cyst (SEAC) is complete surgical removal of cyst and closure of the dural defect. In most cases, total laminectomy has been performed at affected vertebra for complete removal of the SEAC. However, this invasive surgery may result in postoperative kyphosis and back pain. We report a case of large SEAC involving T10-L1 which was excised through the minimal skipped hemilaminectomy, to minimize the risk of postoperative kyphotic deformity. Simultaneously, we closed the dural defect after preoperative precise identification of the site through several radiological studies.

Key Words: Spinal $\cdot$ Extradural $\cdot$ Arachnoid cyst $\cdot$ Minimal

\section{INTRODUCTION}

Spinal extradural arachnoid cysts (SEACs) are uncommon, accounting for about $1 \%$ of all primary spinal cord tumors and rare expanding lesions in the spinal canal ${ }^{11)}$. They may arise from a congenital dural defect which allows the arachnoid membrane to herniate through the adjacent dura mater, and communicate with the intraspinal subarachnoid space through the defect. Cyst expansion may result from active secretion from internal lining cell, osmotic gradient between the subarachnoid space and cyst, pulsatile cerebrospinal fluid dynamics, and one-way valve mechanism ${ }^{3}$. If symptoms develop due to cyst expansion, surgical treatment is required. The treatment of choice is complete cyst removal and closure of the dural defect ${ }^{2,3,6,9,10)}$. In most cases, total laminectomy was performed at affected vertebra for complete removal of the cyst with or without closing the dural defect ${ }^{2,3,6,9,10)}$. However, this invasive surgery may result in postoperative kyphosis and back pain $^{1,6)}$.

We report a case of large thoracolumbar extradural arach-

- Received: November 30, 2012 • Revised: January 23, 2013

- Accepted: January 28, 2013

Corresponding Author: Won Ho Cho, MD, PhD

Department of Neurosurgery, Pusan National University Hospital, 305

Gudeok-Ro Seo-Gu, Busan 602-739, Korea

Tel: +82-51-240-7257, Fax: +82-51-244-0282

E-mail: mdcwh@naver.com

*This work was supported by clinical research grant from Pusan National University Hospital 2011. noid cyst excised by minimal hemilaminectomy to minimize the risk of postoperative kyphotic deformity, repairing dural defect after precise radiological detection.

\section{CASE REPORT}

A 43-year-old man presented with back pain and radiating pain of both legs for 5 years. The symptoms had developed over last 1 year. Neurologic examination revealed mild voiding difficulty. There was no history of trauma, lumbar puncture, any surgery and spinal infection. Plain radiography and computed tomography (CT) of thoracolumbar spine revealed thinned pedicles, increased diameter of spinal canal, and posterior scalloping of the vertebral bodies at T10 to L1. Magnetic resonance imaging (MRI) demonstrated a large extradural cystic lesion at T10-L1 levels with extension into the foramen and extra-foramen. The cystic lesion was iso-intense compared with CSF on T1 and T2-weighted images and no enhancement after gadolinium administration was showed (Fig. 1). The preoperative diagnosis was spinal extradural arachnoid cyst. Because the closure of dural defect is important for prevention of recurrence, the preoperative radiologic studies were performed to detect the dural defect. First, cine-MRI and magnetic resonance (MR) myelography were performed. We were not able to confirm the communication site by cine-MRI and MR myelography. Additionally, CT myelography was performed. It showed contrast medium leakage at T12 vertebral body lower margin (Fig. 2). This finding provided an evidence of a communication between the extradural cyst and normal 

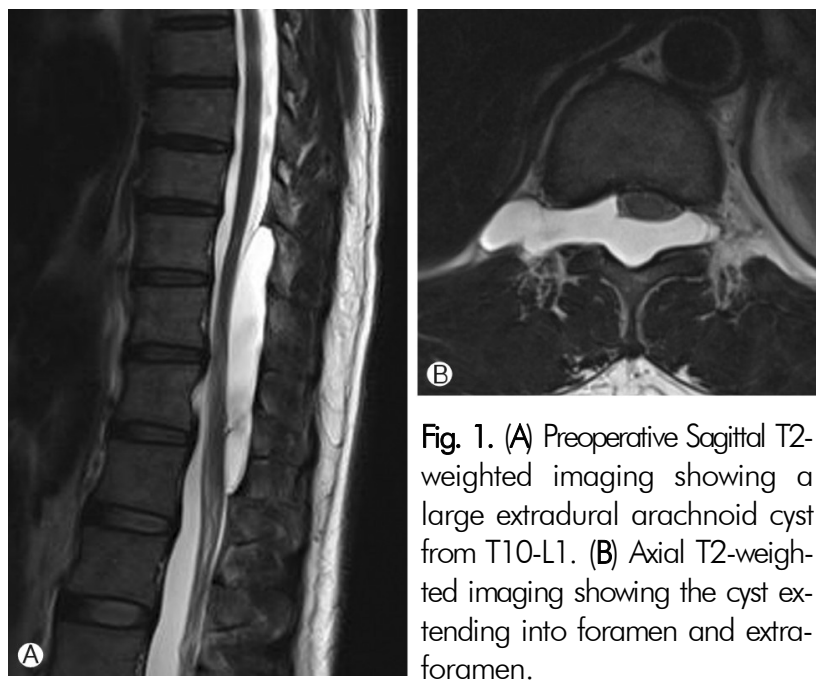

Fig. 1. (A) Preoperative Sagittal T2weighted imaging showing a large extradural arachnoid cyst from T10-L1. (B) Axial T2-weighted imaging showing the cyst extending into foramen and extraforamen.
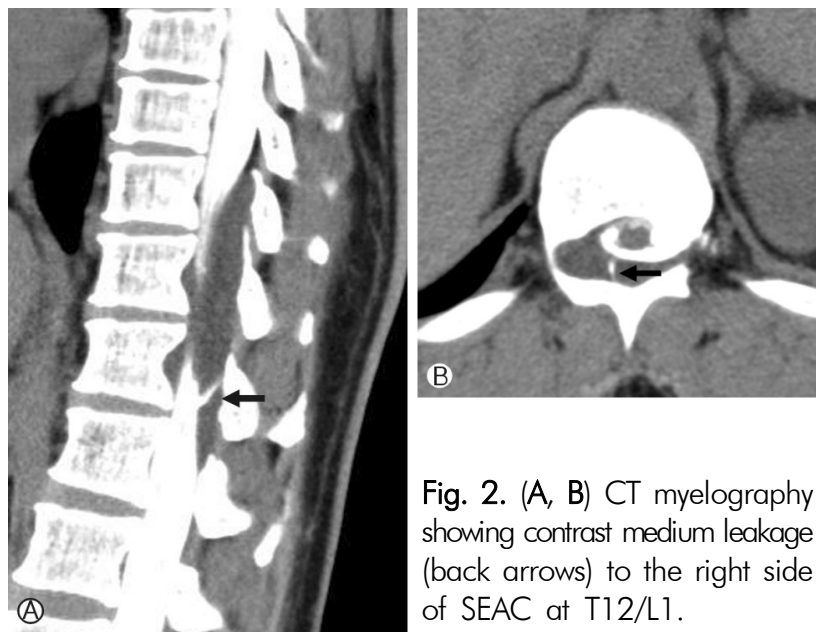

Fig. 2. (A, B) CT myelography showing contrast medium leakage (back arrows) to the right side of SEAC at T12/L1.

subarachnoid space. We could localize the communication site between the cyst and subarachnoid space before the operation.

First of all, right hemilaminectomy was performed from low part of T11-upper part of L1 for exploration of the communication site. A huge epidural arachnoid cyst was exposed and outer wall was incised. The yellowish cyst fluid was expelled. The cystic mass was easily separated from the dorsal thecal sac. Then, additional hemilaminectmy was performed at T10 and upper portion of T11, leaving two third of T11 lamina. The upper margin of the cyst was exposed and separated from the dura sac. Without partial resection, the separated upper part of the cyst was passed beneath L1 lamina and continually separated. But, cystic wall extending to right foramen of T11/12 was not separated due to adhesion with surrounding tissue. So, the cyst was excised, leaving small remnant at right foramen of T11/12. After removing the cyst, we found a small dural defect and CSF leak at the dorsal thecal sac at T12/L1. After repairing the defect with primary
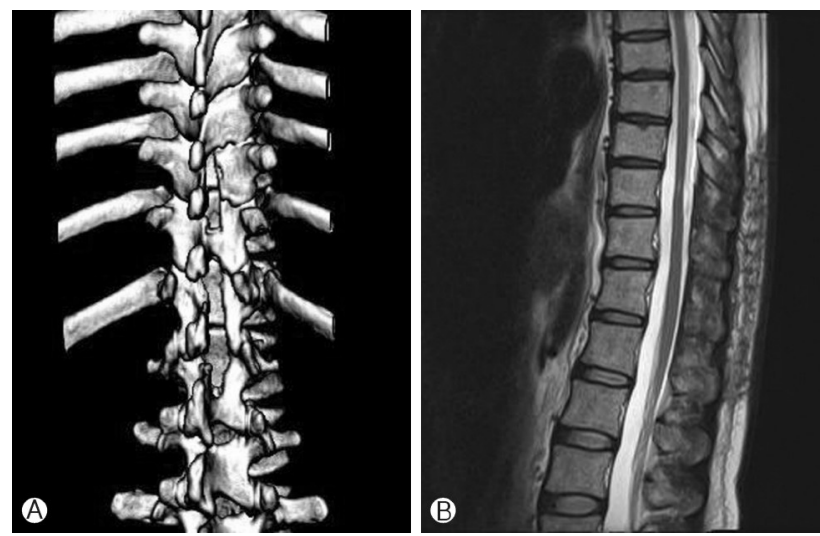

Fig. 3. (A) Postoperative 3-dimentional CT showing the right skipped hemilaminectomy leaving right $\mathrm{T} 11$. (B) Follow-up MRI showing no recurrence of the arachnoid cyst.

suture, the site was sealed using fibrin glue. There were no post-operative complications. The patient's pain improved rapidly. After 10 days, the patient was discharged. At 1 year after the operation follow up, CT and MRI demonstrated no recurrence (Fig. 3). Dynamic plain X-ray revealed no instability and kyphotic deformity.

\section{DISCUSSION}

For incidental SEAC with no symptoms, surgery is not recommended. However, if progressive neurologic dysfunction appears due to compression of the spinal cord or nerve roots, surgical treatment is necessary ${ }^{4)}$.

Several surgical methods for SEAC have been proposed. The standard treatment for symptomatic spinal epidural arachnoid cyst is complete surgical removal of cyst $^{2-4,7,9,10)}$. However, all SEACs cannot be completely excised due to foraminal or extraforaminal extension or extensive adhesion of the cyst to dural $\mathrm{sac}^{4,8}$. If the cyst is adherent to the dural sac, marsupialization could relieve the symptoms. Simple aspiration and evacuation of the cyst relieve only temporarily symptoms ${ }^{10)}$. A selective interlaminar fenetration at the transdural communication site with clipping of the dural rent, following preoperative detection of communication site, has been reported to result in cyst regression and good clinical course ${ }^{8)}$. One report describes an expansive duroplasty technique, which sutures cyst to opened dura and creates an expanded dural sac with single lumen, achieved complete resolution of the symptoms ${ }^{5)}$.

Among several treatment modalities, complete resection of the cyst wall with laminectomy has been frequently performed. Even if conventional laminectomy can provide a sufficient exposure of large cyst, there could be several postope- 
rative complication including postoperative hematoma, scar formation, postoperative instability, and spinal deformity of the spine. Especially, the incidence of kyphotic deformity after conventional laminectomy is reported $33 \%$ to $100 \%{ }^{1)}$. To prevent these risks, laminoplasty has been recommended for lesions of the thoracic and lumbar spine, ${ }^{5,6}$. Also, in several reports, SEACs have been excised through a laminoplasty. However, laminoplasty cannot provide good intraoperative visualization and total cyst removal is difficult, especially in large cyst extending into foraminal or extraforaminal portion. Miyakoshi et al. excised totally a huge SEAC extending into the neural formina by recapping T-saw laminoplasty, preserving complete posterior spinal elements ${ }^{6}$.

Nevertheless, there is still a controversy for the necessity of total cyst resection through conventional laminectomy or laminoplasty due to the risks of postoperative kyphotic deformity, longer operative time, and technical difficulty. In addition, closure of the dural defect without cyst resection has been reported to achieve good neurological recovery without recurrence $^{8}$. Considering the pros and cons of two main surgical options, we decided to remove cyst wall as much as possible by minimal skipped hemilaminectomy and close the dural defect for the treatment of the large SEAC. Through the minimal skipped hemilaminectomy, we were able to remove the arachnoid cyst near-totally, leaving small remnant of cyst wall at right T10/11 foramen and close the dural defect at the same time. The minimal skipped hemilaminectomy can minimize the risks of postoperative instability by preserving opposite muscular and bony structures. Skipping of hemilaminec- tomy enables excision of a huge extradural arachnoid cyst involving multiple segments and interrupted intact lamina minimize the postoperative instability. In addition, the hemilaminectomy can provide enough intra-operative visualization of lesion and working space, to close the dural defect. However, if the SEAC adheres to the dural sac and nerve root or extend into foramen or extraforaminal portion, enough resection of cystic wall could be difficult. Therefore, preoperative detection of the dural defect is important in choosing the skipped hemilaminectomy because closure of dural defect contributes to prevention of recur rence.

Generally, CT myelography has been used as a sensitive diagnostic tool to detect the dural defect ${ }^{4}$. However, the preoperative identification of dural defect could be difficult. Neo et al. diagnosed the location of the communication site using cine-MRI detect and selectively closed the dural defect ${ }^{8)}$. The communication site on the cine-MRI was showed as a pulsating flow void. Miyamoto et al. suggested a preoperative MRI myelography is helpful for detecting the dural in a patient with a SEAC ${ }^{7}$. In our case, we took CT myelography, MRI myelography, and cine MRI to detect the dural defect. Among several imaging studies, CT myelography definitely showed the dural defect with contrast leakage into the cyst. Reviewing the diagnostic images, there was a difficulty to identify a definite pulsating flow void. Instead, MR myelography showed the finding of communication in accord with the CT myelography. The routine MRI also showed the dural defect as a small spot in the same site. Especially, the dural defect was showed a spot of high-signal intensity on T1-weighted images. Therefore, the routine MRI could be helpful in detecting the dural defect in limited cases.

\section{CONCLUSION}

We report a case of large thoracolumbar extradural arachnoid cyst excised by minimal skipped hemilaminectomy, repairing dural defect after precise radiological detection. We suggested that the minimal skipped hemilaminectomy can be used as a feasible technique for the treatment of large SEACs, removing the cyst wall as much as possible and preventing the postoperative instability. The closure of the dural defect can improve the usefulness of the technique. Especially, CT myelography may be the surest imaging technique for preoperative detection of the dural defect.

Cervical spondylolysis is a very rare condition. Its clinical importance is vulnerability to trauma. The etiology of cervical spondylolysis is still a controversy. Computer tomography and plain film are useful diagnostic image modality.

For whatever reasons, symptomatic patients need to be treated by conservative or surgical option. Giving more attention to cervical spondylolysis will help further exact diagnosis and appropriate therapy.

\section{REFERENCES}

1. Amhaz HH, Fox BD, Johnson KK, Whitehead WE, Curry DJ, Luerssen TG, et al: Postlaminoplasty kyphotic deformity in the thoracic spine: case report and review of the literature. Pediatr Neurosurg 45:151-154, 2009

2. Bae JS, Choi JY, Kim SH, Sung KH: Extradural arachnoid cyst in the thoracic spine. Korean J Spine 2:172-175, 2005

3. Choi JY, Kim SH, Lee WS, Sung KH: Spinal extradural arachnoid cyst. Acta Neurochir (Wien) 148:579-585; discussion 585, 2006

4. Hughes G, Ugokwe K, Benzel EC: A review of spinal arachnoid cysts. Cleve Clin J Med 75:311-315, 2008

5. Kikuta K, Hojo M, Gomi M, Hashimoto N, Nozaki K: Expansive duraplasty for the treatment of spinal extradural arachnoid cysts: case report. J Neurosurg Spine 4:251-255, 2006

6. Miyakoshi N, Hongo M, Kasukawa Y, Shimada Y: Huge thoracolumbar extradural arachnoid cyst excised by recapping Tsaw laminoplasty. Spine J 10:e14-18, 2010 
7. Miyamoto M, Kim K, Matsumoto R, Isobe M, Isu T: Utility of preoperative magnetic resonance imaging myelography for identifying dural defects in patients with spinal extradural arachnoid cysts: case report. Neurosurgery 59:E941; discussion E941, 2006

8. Neo M, Koyama T, Sakamoto T, Fujibayashi S, Nakamura T: Detection of a dural defect by cinematic magnetic resonance imaging and its selective closure as a treatment for a spinal extra- dural arachnoid cyst. Spine (Phila Pa 1976) 29:E426-430, 2004

9. Payer M, Bruhlhart K: Spinal extradural arachnoid cyst: review of surgical techniques. J Clin Neurosci 18:559-560, 2011

10. Tureyen K, Senol N, Sahin B, Karahan N: Spinal extradural arachnoid cyst. Spine J 9:e10-15, 2009

11. Yabuki S, Kikuchi S: Multiple extradural arachnoid cysts: report of two operated cousin cases. Spine (Phila Pa 1976) 32:E585588, 2007 